\title{
Primary Hepatoid Adenocarcinoma of the Lung
}

\author{
${ }^{1}$ Gnanapriya Vellaisamy, ${ }^{2}$ Pritilata Rout, ${ }^{3}$ Julian Crasta, ${ }^{4}$ Uma Devaraj
}

\begin{abstract}
Hepatoid adenocarcinoma lung ( $\mathrm{HAL})$ is an extremely rare type of extrahepatic adenocarcinoma with morphological similarity to hepatocellular carcinoma. We report a case of 59 years male smoker who presented with anorexia, weight loss and cough. Chest X-ray revealed a homogenous opacity in the left upper and mid zone. Computed tomography (CT) chest showed a left upper lobe mass with enlarged hilar lymph nodes. Serum alpha fetoprotein (AFP) was normal. Guided fine needle aspiration cytology (FNAC) of the mass revealed a few atypical cells. Endobronchial biopsy showed sheets of polygonal cells with coarsely clumped chromatin, eosinophilic nucleoli and abundant eosinophilic to clear cytoplasm. The neoplastic cells were positive for hepatocyte-1, Carcinogenic embryonic antigen (CEA), Cytokeratin 7 (CK-7) and cytoplasmic positivity for thyroid transcription factor (TTF-1). After 3 months, the tumor had grown significantly. Due to its aggressive behavior, early and accurate diagnosis is necessary.
\end{abstract}

Keywords: Adenocarcinoma, Hepatoid, Pulmonary origin

How to cite this article: Vellaisamy G, Rout P, Crasta J, Devaraj U Primary Hepatoid Adenocarcinoma of the Lung. J Postgrad Med Edu Res 2016;50(2):103-106.

Source of support: Nil

Conflict of interest: None

\section{INTRODUCTION}

Hepatoid adenocarcioma is an extremely rare type of tumor with its histological resemblance to hepatocellular carcinoma. Stomach is the most common site, whereas lung accounts for nearly $5 \%$ of its incidence. ${ }^{1}$ We describe a case of hepatoid carcinoma arising from the lung. It is generally associated with a poor prognosis.

\section{CASE REPORT}

A 59-year-male ex-smoker presented with complaints of anorexia and weight loss for 6 months, low grade fever for 3 weeks and cough for 10 days. On examination his pulse rate was $84 /$ minute and blood pressure was $150 / 80 \mathrm{~mm} \mathrm{Hg}$. Respiratory examination revealed

\footnotetext{
${ }^{1}$ Lecturer, ${ }^{2}$ Professor and Head

${ }^{3}$ Additional Professor, ${ }^{4}$ Associate Professor

${ }^{1-3}$ Department of Pathology, St Johns Medical College, Bengaluru Karnataka, India

${ }^{4}$ Department of Chest Medicine, St Johns Medical College and Hospital, Bengaluru, Karnataka, India

Corresponding Author: Gnanapriya Vellaisamy, Lecturer Department of Pathology, St Johns Medical College, Bengaluru Karnataka, India, Phone: 08022065008, e-mail: drvgnanapriya@ gmail.com
}

decreased breath sounds, impaired note on percussion and crepitations in right infraclavicular area. Rest of the systemic examinations was largely unremarkable. Lab investigations including hemoglobin, total count, platelet count, prothrombin, and activated prothrombin time were within the normal range. Serum alpha-fetoprotein (AFP) was normal $(0.8 \mathrm{ng} / \mathrm{ml})$. A homogenous opacity was noted in the left upper and mid zone in the chest X-ray. Computed tomography (CT) chest revealed a large solid heterogeneously enhancing mass lesion occupying the left upper lobe measuring $13 \times 9.4 \times 7.5 \mathrm{~cm}$ with multiple enlarged subcarinal lymphnodes largest measuring $2.7 \mathrm{~cm}$ (Fig. 1) and a solitary well-defined homogenously enhan-cing benign cystic lesion without solid areas and necrosis in the liver measuring $1.2 \mathrm{~cm}$. Clinical stage was stage IIIA (cT3N2M0). On bronchoscopy, left upper bronchus was completely occluded by intrabronchial growth. He underwent image-guided fine needle aspiration cytology (FNAC) of the mass which revealed a few clusters of atypical cells with abundant eosinophilic cytoplasm (Fig. 2). Endobronchial biopsy showed an infiltrating neoplasm composed of sheets of polygonal cells with distinct cell borders exhibiting round nuclei, coarsely clumped chromatin, prominent eosinophilic nucleoli, abundant eosinophilic to clear cytoplasm (Fig. 3). Both intra and extracellular periodic acid-Schiff (PAS) positive diastase resistant hyaline globules were noted in few of the cells. Bile plugs were absent. Amidst these cells, there were areas of necrosis. In view of hepatoid morphology, immunohistochemical stains were done. The neoplastic cells were positive for cytokeratin 7 (CK7), monoclonal carcinogenic embryonic antigen (CEA), hepatocyte-1, AFP, cytoplasmic positivity for thyroid transcription factor (TTF-1) and negative for CK20, P63, CK5/ 6 and CDX-2 (Figs 4 to 8). A final diagnosis of hepatoid adenocarcinoma of lung was made.

\section{DISCUSSION}

In 1985, Ishikura et al first described hepatoid adenocarcinoma of gastric origin. ${ }^{2}$ In 1990, he described its origin from the lung. He adopted two criteria for diagnosing it:

- A mixture of tubular or papillary adenocarcinoma with sheets of neoplastic cells

- Neoplastic cells with centrally located nuclei, abundant eosinophilic cytoplasm morphologically resembling hepatocellular carcinoma cells. ${ }^{3}$ Our case fulfills the above criteria. 


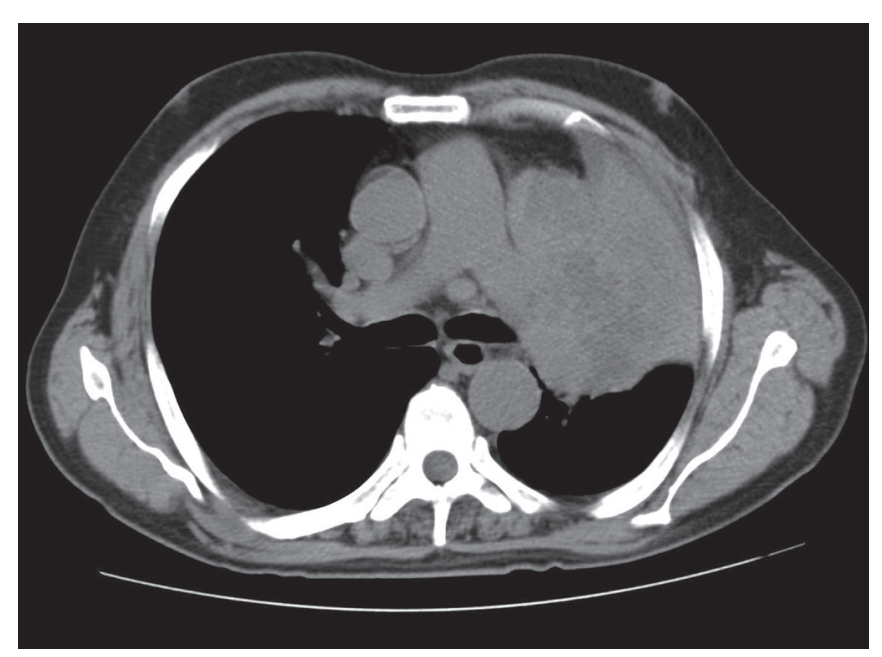

Fig. 1: Computed tomography chest showing a large solid heterogeneously enhancing lesion in the left upper lobe

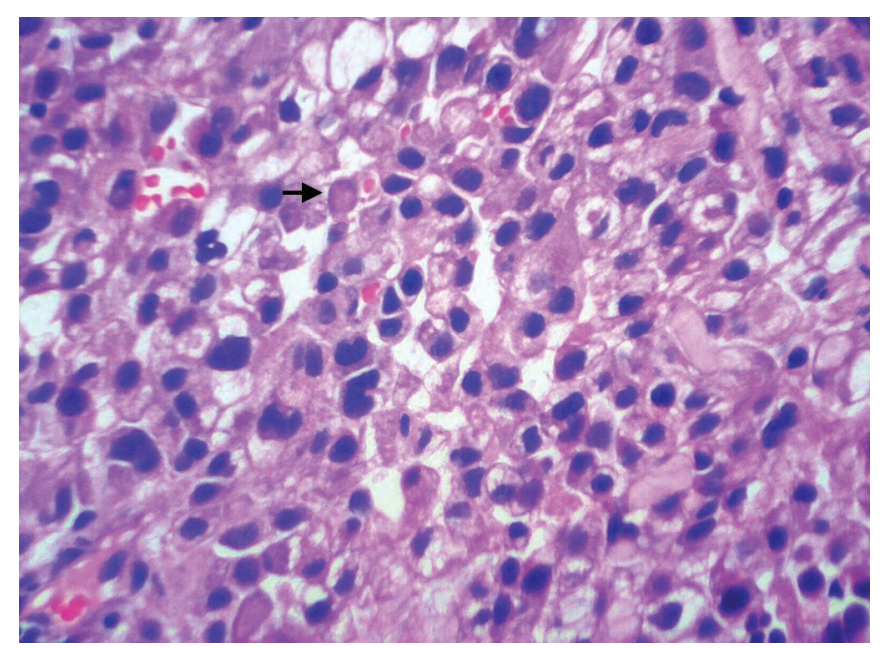

Fig. 3: Biopsy showing sheets of cells with hyaline globules resembling hepatocellular carcinoma cells (arrow mark (hematoxylin and eosin stain. Original magnification 40x)

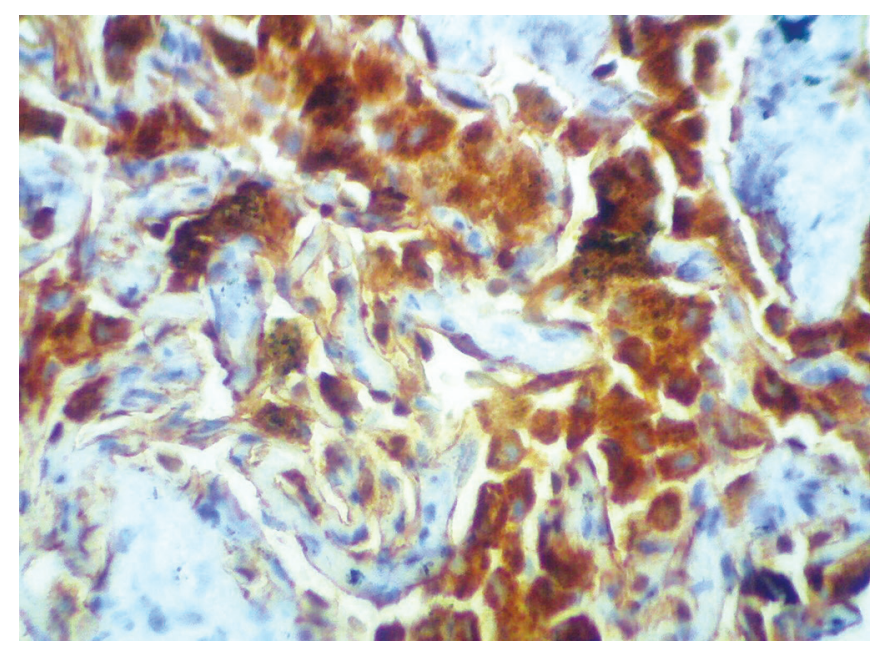

Fig. 5: Monoclonal CEA showing cytoplasmic positivity in the neoplastic cells (original magnification 40x)

The most common location is the stomach (63\%). The other sites of origin include ovary $(10 \%)$, gallbladder $(4 \%)$, pancreas $(4 \%)$, and uterus $(4 \%)$, whereas lung

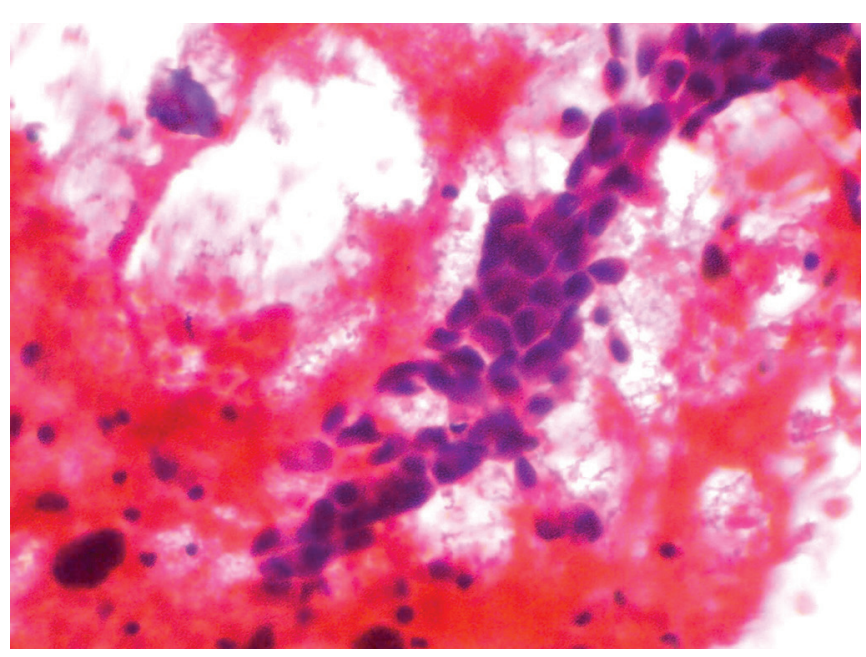

Fig. 2: Fine needle aspiration cytology of the mass showing atypical cells with eosinophilic cytoplasm (hematoxylin and eosin stain. Original magnification 40x)

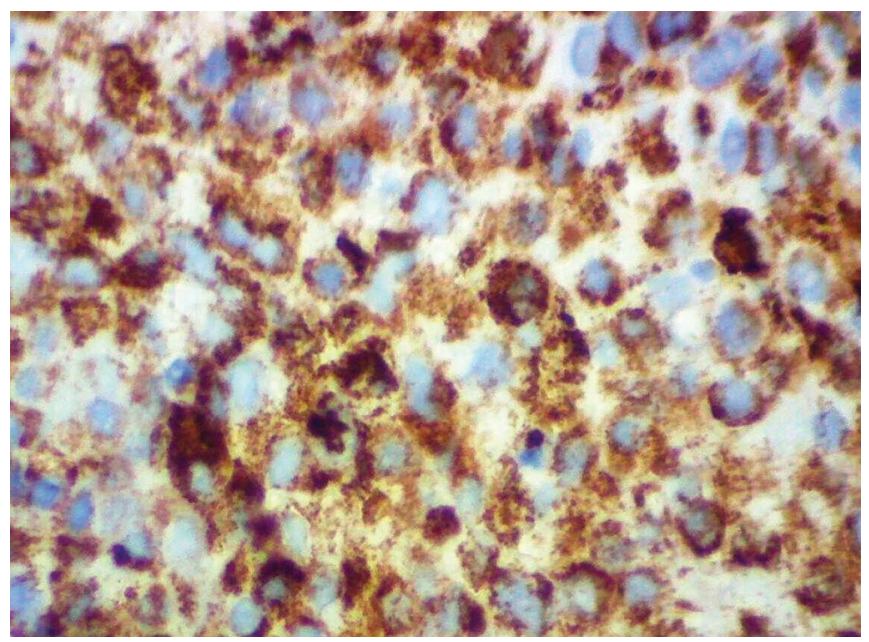

Fig. 4: Hepatocyte-1 showing diffuse cytoplasmic positivity in the neoplastic cells (original magnification 40x)

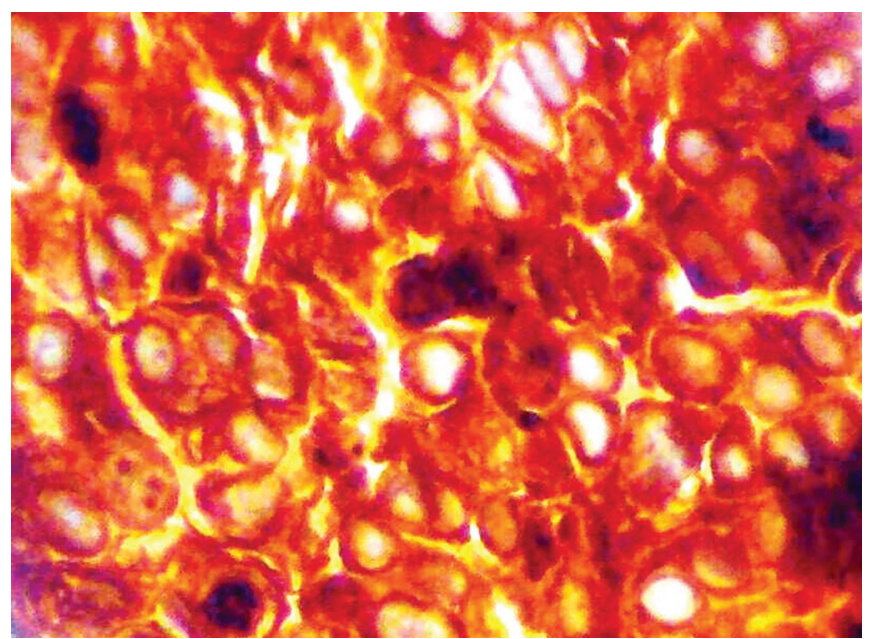

Fig. 6: Cytokeratin 7 showing diffuse cytoplasmic and membranous positivity in the neoplastic cells (original magnification 40x)

accounts for only $5 \%$ of its incidence. ${ }^{1}$ Twenty-two cases were reported in the world literature. ${ }^{4-19}$ Among them, 21 were males and nine were heavy smokers. Majority 


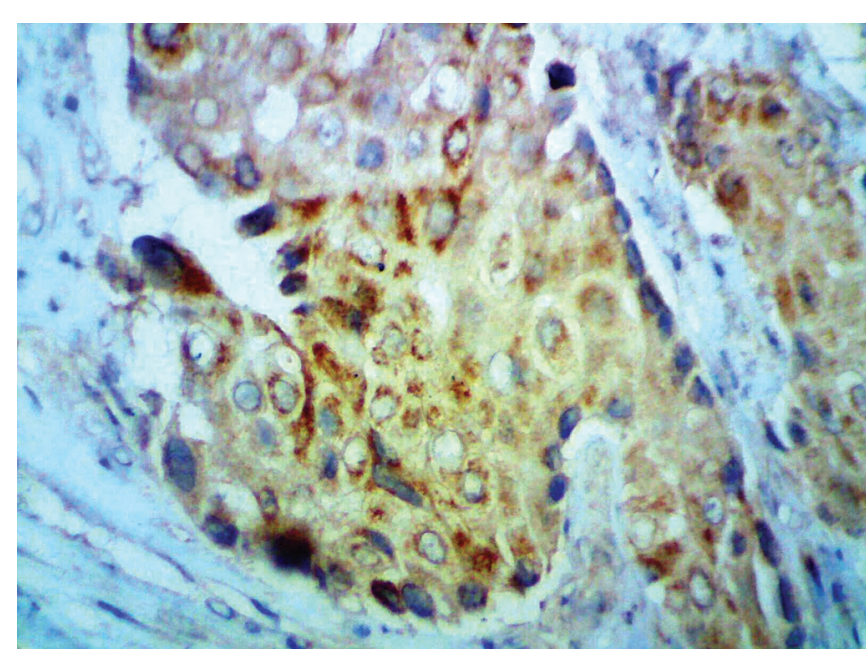

Fig. 7: Thyroid transcription factor-1 showing cytoplasmic positivity in the neoplastic cells (original magnification 40x)

of the cases presented at an advanced stage. The clinical features of our case are similar to the reported cases.

Alpha-fetoprotein is produced by yolk sac and fetal liver. It is generally raised in hepatocellular carcinomas and yolk sac tumors and been used as tumor marker for relapse/recurrence. Hepatoid adenocarcinoma is one of the AFP producing lung tumors. The other lung tumors which produce AFP include intrapulmonary yolk sac tumor and pneumoblastoma. Majority of the reported cases had elevated AFP but in our case, the level was normal. However, tumor cells expressed AFP on immunohistochemistry indicating hepatoid differentiation.

Histologically, the neoplastic cells will be arranged in tubular, papillary, sheets or in trabecular pattern. Periodic acid-Schiff positive diastase resistant hyaline globules is one of the characteristic feature but not specific. These globules may or may not be membrane bound. Type I globules are membrane bound and are seen in the intracytoplasmic lumina, whereas type II globules are seen both intracellularly and extracellularly. Some of these intracytoplasmic globules have peripheral halo with spikes and are called as spicular bodies. Bile plugs may or may not be present. Intracytoplasmic lumina may be seen in a few cells. Sheet like proliferation of neoplastic cells with type II hyaline globules were noted in this case. Before diagnosing a case of primary hepatoid adenocarcinoma lung, it is necessary to exclude metastasis from liver, ovary or stomach. In our case, a cystic lesion was identified in the liver but radiologically, the cyst was benign. Rest of the organs did not show any evidence of metastasis. Immunohistochemically, these cells show positivity for markers of hepatocyte differentiation, such as Hepar-1, AFP, cytoplasmic positivity for TTF-1. In addition, the cells express positivity for markers of adenocarcinoma, such as CK7, monoclonal CEA, Lung carcinoma antibody (MOC31), and epithelial cell adhesion molecule (EPCAM).

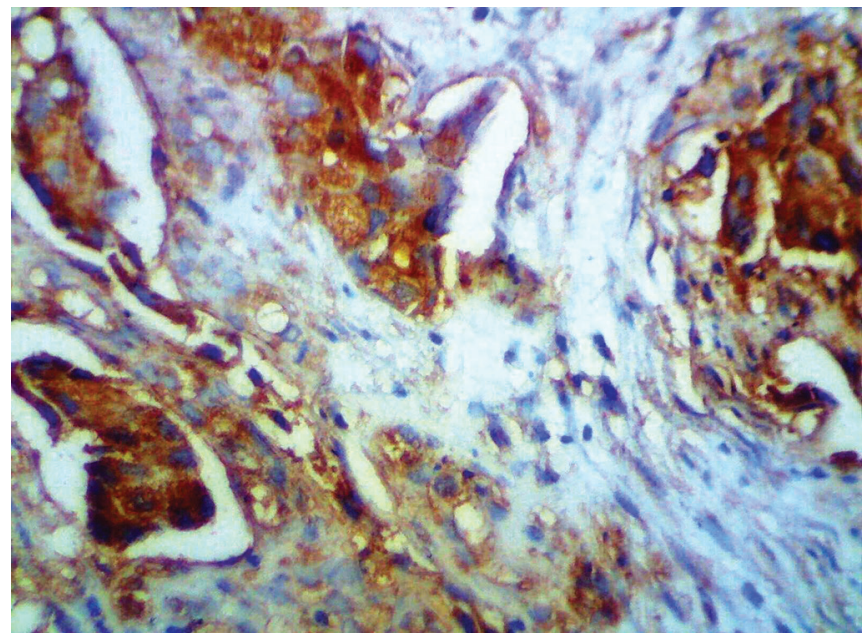

Fig. 8: Alpha-fetoprotein showing diffuse cytoplasmic positivity in the neoplastic cells (original magnification 40x)

Histogenetically, these tumors may arise from ectopic liver, germ cells in the lung or from the multipotential respiratory epithelial cells. ${ }^{3}$ The prognosis is usually worse as majority of these tumors present at an advanced stage. If the diagnosis is made at an early stage, it may be possible to resect the lesion which may improve the prognosis. Our case was staged as IIIA. The patient was offered chemotherapy/radiotherapy which he refused due to financial constraints. After 3 months a follow-up chest X-ray revealed marked increase in size of the homogenous opacity. Hence, the prognosis is expected to be worse in this patient.

\section{CONCLUSION}

Primary hepatoid carcinoma being rare in the lung should be diagnosed after ruling out metastasis from other organs. Recognition of the variant is important due to its aggressive behavior, hence warrants early diagnosis and treatment.

\section{REFERENCES}

1. Metzgeroth G, Strobel P, Baumbusch T, et al. Hepatoid adenocarcinoma: review of the literature illustrated by a rare case originating in the peritoneal cavity. Onkologie 2010;33(5): 263-269.

2. Ishikura H, Fukasawa Y, Ogasawara K, et al. An AFP-producing gastric carcinoma with features of hepatic differentiation: case report. Cancer 1985;56(4):840-848.

3. Ishikura H, Kanda M, Ito M, et al. Hepatoid adenocarcinoma: a distinctive histological subtype of alpha-fetoprotein producing lung carcinoma. Virchows Archiv A Pathol Anat Histopathol 1990;417(1):73-80.

4. Yokoyama K, Morimoto H, Kaito T, et al. An autopsied case of alpha-fetoprotein (AFP) producing large cell carcinoma of the lung. Jpn J Thorac Surg 1981;34(8):609-612.

5. Yasunami R, Hashimoto Z, Ogura T, et al. Primary lung cancer producing alpha-fetoprotein: a case report. Cancer 1981;47(5): 926-929. 
6. Miyake M, Ito M, Taki T, et al. A case report of two patients with primary lung cancer secreting AFP. J Jpn Assoc Thorac Surg 1986;34(6):914-919.

7. Miyake M, Ito M, Mitsuoka, et al. Alpha-fetoprotein and human chorionic gonadotropin-producing lung cancer. Cancer 1987;59(2):227-232.

8. Okunaka T, Kato H, Konaka C, et al. Primary lung cancer producing $\alpha$-fetoprotein. Ann Thorac Surg 1992;53(1):151-152.

9. Nasu M, Soma T, Fukushima H, et al. Hepatoid carcinoma of the lung with production of $\alpha$-fetoprotein and abnormal prothrombin: an autopsy case report. Mod Pathol 1997;10(10): 1054-1058.

10. Arnould L, Drouot F, fargeot $\mathrm{P}$, et al. Hepatoid adenocarcinoma of the lung: report of a case of an unusual alpha-fetoproteinproducing lung tumor. Am J Surg Pathol 1997;21(9):1113-1118.

11. Carlinfante G, Pia Foschini M, Pasquinelli G, et al. Hepatoid adenocarcinoma of the lung: a case report with immunohistochemical, ultrastructural and in situ hybridization findings. Histopathology 2000;37(1):85-94.

12. Hiroshima K, Iyoda A, Toyozaki T, et al. Alpha-fetoproteinproducing lung carcinoma: report of three cases. Pathol Int 2002;52(1):46-53.
13. Hayashi $Y$, Takanashi $Y$, Ohsawa H, et al. Hepatoid adenocarcinoma in the lung. Lung Cancer 2002;38(2):211-214.

14. Genova S. Hepatoid adenocarcinoma of the lung: a case report. Internet J Pathol 2002;2(1):1-5.

15. Iino K, Ohta $Y$, Tamura $\mathrm{M}$, et al. A resected case of alphafetoprotein producing. Hepatoid adenocarcinoma of the lung. Jpn I Lung Cancer 2003;43(1):29-34.

16. Oshiro Y, Takada Y, Enomoto T, et al. A resected case of metachronous liver metastasis from lung cancer producing alpha-fetoprotein (AFP) and protein induced by vitamin K absence or antagonist II (PIVKAII). Hepatogastroenterology 2004;51(58):1144-1147.

17. Kishimoto T, Yano T, Hiroshima K, et al. A case of $\alpha$-fetoprotein producing pulmonary carcinoma with restricted expression of hepatocyte nuclear factor-4a in hepatoid foci: a case report with studies of previous cases. Hum Pathol 2008;39(7): 1115-1120.

18. Kim L, Song JY, Jin $X$, et al. Hepatoid adenocarcinoma arising in the lung: a case report. Bas Appl Path 2009;2(1):A47.

19. Haninger DM, Kloecker GH, Bousamra M, et al. Hepatoid adenocarcinoma of the lung: report of five cases and review of the literature. Modern Pathology 2014;27(4):535-542. 